\title{
Behavior of the reflection function of a plane-parallel medium for directions of incidence and reflection tending to horizontal directions
}

\author{
J. W. Hovenier ${ }^{1}$ and D. M. Stam ${ }^{2,3}$ \\ 1 Astronomical Institute "Anton Pannekoek”, University of Amsterdam, Kruislaan 403, 1098 SJ Amsterdam, The Netherlands \\ e-mail: J.W.Hovenier@uva.nl \\ 2 DEOS, Department of Aerospace Engineering, TU Delft, Kluyverweg 1, 2629 HS, Delft, The Netherlands \\ 3 SRON Netherlands Institute for Space Research, Sorbonnelaan 2, 3584 CA Utrecht, The Netherlands
}

Received 12 August 2008 / Accepted 7 September 2008

\begin{abstract}
The atmospheres of (exo) planets and moons, as well as reflection nebulae, contain in general independently scattering particles in random orientation and are often supposed to be plane-parallel. Relations are presented for the (bidirectional) reflection function and several related functions of such a medium in case the directions of incidence and reflection both tend to horizontal directions. The results are quite general. The medium may be semi-infinite or finite, with or without a reflecting surface underneath, and vertically homogeneous or inhomogeneous. Some approximative formulae for the reflection function of a plane-parallel medium with independently scattering particles in random orientation, including Lambert's law, may be very inaccurate if the directions of incidence and reflection are both nearly horizontal.
\end{abstract}

Key words. planets and satellites: general - scattering - radiative transfer - reflection nebulae

\section{Introduction}

A well-known subject in astrophysics concerns multiple scattering of (electromagnetic) radiation in an extended medium containing small, independently scattering particles (see e.g. Ambarzumian 1943; Chandrasekhar 1950; Sobolev 1975; van de Hulst 1980; Hansen \& Travis 1974; Hovenier et al. 2004; Mishchenko et al. 2006). Examples of such media are provided by the atmospheres of (exo)planets and satellites, as well as reflection nebulae and protoplanetary disks. The medium is often supposed to be locally plane-parallel, so that one can use the theory developed for a plane-parallel atmosphere, i.e. a horizontally homogeneous atmosphere of infinite horizontal extent. The radiation (which we will also call light) coming from a distant source, like the Sun or a star, may illuminate the top of the atmosphere and then be scattered by the particles inside before leaving the atmosphere at the top in all upward directions. This is called reflected radiation and, neglecting polarization, the angular distribution of its (specific) intensity can be expressed by means of the so-called reflection function.

The reflection function is an important fundamental property of an atmosphere, normalized so that it is identically equal to one for a perfectly white surface following Lambert's law. Once the reflection function of an atmosphere has been obtained, one readily finds the angular distribution of the intensity of the reflected radiation for any angular distribution of incident radiation at the top. In the literature this function has a variety of names, such as reflection coefficient (Sobolev 1975; Yanovitskij 1997), bidirectional reflection function (Mishchenko et al. 1999) and reflectance factor (Hapke 1993).
Numerous theoretical and numerical studies of reflection functions have been reported in books and papers. Yet, very little attention was given to the limiting case when the directions of incident and reflected radiation both tend to horizontal directions. This case not only provides more insight into the angular distribution of the reflected radiation, but it is also important for exact and approximate computations, in particular when discontinuities are involved. This was shown for the intensity of the reflected radiation, first when polarization is neglected (Hovenier \& Stam 2006) and later when it is taken into account (Hovenier \& Stam 2007). As far as the reflection function is concerned, an interesting statement was made by van de Hulst (1980), namely that the reflection function becomes infinitely large for horizontal directions of both incidence and reflection. But he restricted himself to the azimuth-independent terms in a Fourier series expansion and he did not give any evidence or clarification regarding this statement.

The principal aim of this paper is to present a comprehensive treatment of the behavior of the reflection function and related functions when the directions of incidence and reflection both tend to horizontal directions. The organization of this paper is as follows. Some basic concepts and definitions are discussed in Sect. 2. In Sect. 3, the limiting process of directions of incidence and reflection tending to horizontal directions, while keeping the azimuth difference of the directions fixed, is considered for, respectively, the reflected intensity, the reflection function and orders of scattering of both. Simple examples are given in Sect. 4 for vertically homogeneous as well as inhomogeneous media. Section 5 is devoted to functions that are related to the reflection function. The azimuth dependence of the incident and 
reflected light is treated in Sect. 6. Approximations are discussed in Sect. 7 and some concluding remarks are presented in Sect. 8.

\section{Concepts and defintions}

We consider a plane-parallel atmosphere composed of randomly oriented particles, which may include gas molecules. The particles scatter radiation independently and without change of wavelength in all directions with a scattering angle distribution called the phase function. Since we are mainly interested in natural particles and physically realistic model particles we will assume that the albedo of single scattering is positive but not larger than one and the phase function is a positive, bounded and continuous function of directions. It is normalized so that its average over all directions equals unity.

There are no internal sources in the atmosphere. A parallel beam of radiation, coming from a distant source, is incident on each point of the top of the atmosphere. The net flux per unit area normal to this beam is $\pi F_{0}$. The direction of the incident beam is given by $\mu_{0}$, which is the cosine of the angle this direction makes with the downward normal, and an azimuthal angle $\phi_{0}$. Polarization is ignored and we focus on the reflected radiation, i.e. the radiation that emerges at the top of the atmosphere. Its direction is described by $\mu$, the cosine of the angle this direction makes with the upward normal, and an azimuthal angle $\phi$. The azimuthal angles are measured from an arbitrary zero direction in an arbitrary sense and only the difference, $\phi-\phi_{0}$, is relevant, since the medium is horizontally homogeneous. It should be noted that with our definitions $\mu$ and $\mu_{0}$ are non-negative and we have $0 \leq \phi-\phi_{0} \leq 2 \pi$. In this paper we are mainly interested in the question what happens with the reflection properties of the atmosphere if $\mu$ and $\mu_{0}$ both approach zero, starting from values larger than zero. form

The intensity of the reflected radiation can be written in the

$I^{\mathrm{t}}\left(\mu, \mu_{0}, \phi-\phi_{0}\right)=\mu_{0} R\left(\mu, \mu_{0}, \phi-\phi_{0}\right) F_{0}$.

Here and hereafter the superscript $\mathrm{t}$ is used to indicate the top of the atmosphere and $R\left(\mu, \mu_{0}, \phi-\phi_{0}\right)$ is the reflection function. The intensity at the top and the reflection function are both nonnegative. Once the reflection function has been obtained one readily finds the intensity of the reflected radiation for a parallel beam of incident radiation by using Eq. (1), while for multi-directional incident light an integration over all incident directions must be performed.

\section{Approaching the origin in the $\left(\mu_{0}, \mu\right)$-plane}

A function of one real variable may have two different limits at a point of the real number axis, namely a right-hand limit and a left-hand limit. For a function of two or more real variables there are many more possibilities to approach a point in the relevant multi-dimensional space and this may or may not correspond to a number of different values to which the function approaches (Courant 1962). This will be considered in the following sections for, respectively, the intensity of the reflected radiation, the reflection function and orders of scattering of both.

\subsection{Intensity of the reflected radiation}

Let us keep the azimuth difference fixed so that the functions $I^{\mathrm{t}}\left(\mu, \mu_{0}, \phi-\phi_{0}\right)$ and $R\left(\mu, \mu_{0}, \phi-\phi_{0}\right)$ are functions of the two variables, $\mu$ and $\mu_{0}$. We can approach the point $\mu=\mu_{0}=0$ in various

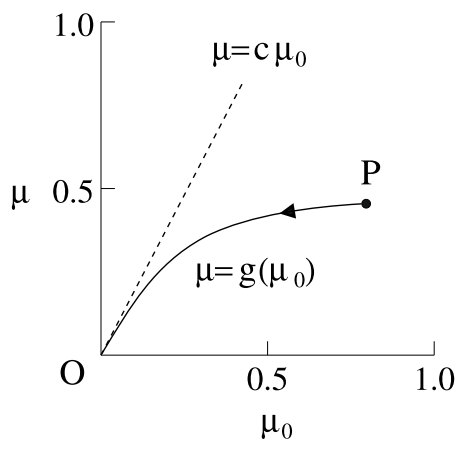

Fig. 1. A point $\mathrm{P}$ approaches the origin $\mathrm{O}$ of a Cartesian coordinate system along a curve (solid line) represented by $\mu=g\left(\mu_{0}\right)$, which has a slope $c$ at $\mathrm{O}$ with respect to the positive $\mu_{0}$-axis. The tangent of the curve at $\mathrm{O}$ has also been drawn (dashed line).

ways. This can be visualized by saying that we can approach the origin, $\mathrm{O}$, in the first quadrant of a Cartesian $\left(\mu_{0}, \mu\right)$-coordinate system by following different paths (curves, including straight lines). Suppose we represent such a curve by means of a continuous function $\mu=g\left(\mu_{0}\right)$ through $\mathrm{O}$ with a definite tangent at $\mathrm{O}$ (see Fig. 1). If we now approach the origin along this curve, the ratio $g\left(\mu_{0}\right) / \mu_{0}$ will tend to the slope of the tangent at $\mathrm{O}$, which we denote as $c$. For a non-perpendicular tangent at $\mathrm{O}$ the value of $c$ is finite and equals the right-hand derivative of $g\left(\mu_{0}\right)$ at O. Evidently, $\mu_{0}$ and $g\left(\mu_{0}\right)$ are both non-negative, so that $c$ is non-negative. If the curve is a straight line we simply have $g\left(\mu_{0}\right)=c \mu_{0}$. If we first let $\mu$ and then $\mu_{0}$ tend to zero we have $c=0$. If we first let $\mu_{0}$ and then $\mu$ approach zero we can treat this case separately or by letting $c$ tend to infinity.

As an illustration we may consider the special case of isotropic scattering in a semi-infinite homogeneous atmosphere with an albedo of single scattering $a$. The phase function is then identically equal to one and we have (Chandrasekhar 1950)

$I_{1}^{\mathrm{t}}\left(\mu, \mu_{0}, \phi-\phi_{0}\right)=\frac{a F_{0}}{4} \frac{\mu_{0}}{\mu+\mu_{0}}$,

where the subscript 1 refers to the first order of scattering. Writing $\lim _{\mu, \mu_{0} \rightarrow 0}$ for the limit if we approach $\mathrm{O}$ along a curve represented by $\mu=g\left(\mu_{0}\right)$ we readily find

$\lim _{\mu, \mu_{0} \rightarrow 0} I_{1}^{\mathrm{t}}\left(\mu, \mu_{0}, \phi-\phi_{0}\right)=\frac{a F_{0}}{4(c+1)}$,

which shows that the result depends on $c$, i.e. on the path that is taken to approach the origin. Consequently, there is a discontinuity for the intensity of the reflected radiation when the directions of incidence and reflection both become horizontal. This was called a peculiar discontinuity by Hovenier \& Stam (2006), since it looks at first glance rather surprising.

In general, the scattering may be anisotropic, the atmosphere may be vertically inhomogeneous and its optical thickness may be finite with a reflecting or totally absorbing surface underneath the atmosphere. The intensity of the reflected radiation in this general case for near-horizontal directions was also considered by Hovenier \& Stam (2006). They found a peculiar discontinuity in this intensity when $\mu$ and $\mu_{0}$ both approach the origin in the $\left(\mu_{0}, \mu\right)$-plane, which can be written as

$$
\begin{aligned}
\lim _{\mu, \mu_{0} \rightarrow 0} I^{\mathrm{t}}\left(\mu, \mu_{0}, \phi-\phi_{0}\right) & =\lim _{\mu, \mu_{0} \rightarrow 0} I_{1}^{\mathrm{t}}\left(\mu, \mu_{0}, \phi-\phi_{0}\right) \\
& =\frac{a^{\mathrm{t}}}{4(c+1)} Z^{\mathrm{t}}\left(\cos \left(\phi-\phi_{0}\right)\right) F_{0},
\end{aligned}
$$


where $a^{\mathrm{t}}$ is the albedo of single scattering at the top of the atmosphere and $Z^{t}(\cos \Theta)$ is the phase function at the top of the atmosphere with scattering angle $\Theta$. Naturally, in Eq. (4) the same path must be followed for the two limits (see Fig. 1). Consequently, the following conclusions can be drawn for the intensity of the reflected radiation in the limit of $\mu$ and $\mu_{0}$ both being zero: a) the optical thickness of the atmosphere and the reflection properties of the underlying surface are irrelevant; $b$ ) the values of the albedo of single scattering and the phase function need only to be known at the top of the atmosphere; c) orders of scattering higher than the first do not contribute; d) for any path with $c$ unequal to infinity the azimuth dependence is proportional to the scattering angle dependence of the phase function at the top. The constant of proportionality becomes zero if $c$ equals infinity. A similar statement was made by Minnaert (1935) for a semi-infinite homogeneous atmosphere, but he did not provide a correct proof and did not mention that the constant of proportionality depends on the way $\mu$ and $\mu_{0}$ tend to zero. For more details about the behavior of the reflected intensity for directions of incidence and reflection that both tend to horizontal directions we refer to Hovenier \& Stam (2006).

\subsection{The reflection function}

Let us now consider what happens with the reflection function on approaching the origin in the $\left(\mu_{0}, \mu\right)$-plane. Combining Eqs. (1) and (4) gives

$$
\begin{aligned}
\lim _{\mu, \mu_{0} \rightarrow 0} \mu_{0} R\left(\mu, \mu_{0}, \phi-\phi_{0}\right) & =\lim _{\mu, \mu_{0} \rightarrow 0} \mu_{0} R_{1}\left(\mu, \mu_{0}, \phi-\phi_{0}\right) \\
& =\frac{a^{\mathrm{t}}}{4(c+1)} Z^{\mathrm{t}}\left(\cos \left(\phi-\phi_{0}\right)\right) .
\end{aligned}
$$

Using the principle of reciprocity (van de Hulst 1980) we have for all orders of scattering and their sum

$R\left(\mu, \mu_{0}, \phi-\phi_{0}\right)=R\left(\mu_{0}, \mu, \phi-\phi_{0}\right)$.

So, interchanging $\mu$ and $\mu_{0}$ in Eq. (5) and taking limits following the same paths as before we find

$$
\begin{aligned}
\lim _{\mu, \mu_{0} \rightarrow 0} \mu R\left(\mu, \mu_{0}, \phi-\phi_{0}\right) & =\lim _{\mu, \mu_{0} \rightarrow 0} \mu R_{1}\left(\mu, \mu_{0}, \phi-\phi_{0}\right) \\
& =\frac{a^{\mathrm{t}} c}{4(c+1)} Z^{\mathrm{t}}\left(\cos \left(\phi-\phi_{0}\right)\right),
\end{aligned}
$$

since $c$ in Eq. (5) had to be replaced by $1 / c$, i.e. the slope of $g\left(\mu_{0}\right)$ at $\mathrm{O}$ with the positive $\mu$-axis. Since $c$ is nonnegative, the limits in Eqs. (5) and (7) are bounded. So it follows from either one that

$\lim _{\mu, \mu_{0} \rightarrow 0} \mu \mu_{0} R\left(\mu, \mu_{0}, \phi-\phi_{0}\right)=0$.

Since the right-hand side of this equation does not depend on $c$, this limit is the same for all curves represented by $\mu=g\left(\mu_{0}\right)$. More limits of this type, i.e. not depending on $c$, will be encountered further down in this paper.

By adding Eqs. (5) and (7), we obtain

$$
\begin{aligned}
\lim _{\mu, \mu_{0} \rightarrow 0}\left(\mu+\mu_{0}\right) R\left(\mu, \mu_{0}, \phi-\phi_{0}\right) & =\lim _{\mu, \mu_{0} \rightarrow 0}\left(\mu+\mu_{0}\right) R_{1}\left(\mu, \mu_{0}, \phi-\phi_{0}\right) \\
& =\frac{a^{\mathrm{t}}}{4} Z^{\mathrm{t}}\left(\cos \left(\phi-\phi_{0}\right)\right) .
\end{aligned}
$$

This result has been reported for the special case of a semiinfinite, homogeneous atmosphere by several authors (see e.g. Sobolev 1975; Mishchenko et al. 2006; Kokhanovsky 2001), but without a rigorous proof.
Since the far right-hand side of Eq. (9) is positive we must have

$\lim _{\mu, \mu_{0} \rightarrow 0} R_{1}\left(\mu, \mu_{0}, \phi-\phi_{0}\right)=\infty$

and

$\lim _{\mu, \mu_{0} \rightarrow 0} R\left(\mu, \mu_{0}, \phi-\phi_{0}\right)=\infty$,

because zero or any finite number for these limits would be in conflict with Eq. (9). Consequently, we have proved that $R_{1}\left(\mu, \mu_{0}, \phi-\phi_{0}\right)$ as well as $R\left(\mu, \mu_{0}, \phi-\phi_{0}\right)$ have a discontinuity if $\mu$ and $\mu_{0}$ are both zero. The nature of these discontinuities is, however, quite different from the peculiar discontinuities for the intensities discussed in Sect. 3.1.

\subsection{Orders of scattering}

The reflected intensity and the reflection function can be written as a sum (series) of nonnegative terms representing orders of scattering (van de Hulst 1980). In view of Eq. (4) we have for the $n$th order of scattering

$$
\lim _{\mu, \mu_{0} \rightarrow 0} I_{n}^{\mathrm{t}}\left(\mu, \mu_{0}, \phi-\phi_{0}\right)=0 \text { for } n>1 \text {. }
$$

However, we cannot infer from Eqs. (10), (11) what will happen with $R_{n}\left(\mu, \mu_{0}, \phi-\phi_{0}\right)$ for $n>1$ if $\mu$ and $\mu_{0}$ both approach zero. The result might be zero, a finite positive number or infinity. However, some interesting properties for the higher orders of scattering of the reflection function can be obtained as follows. Equation (9) shows that

$\lim _{\mu, \mu_{0} \rightarrow 0}\left(\mu+\mu_{0}\right) R_{n}\left(\mu, \mu_{0}, \phi-\phi_{0}\right)=0$ for $n>1$.

Furthermore, writing

$\frac{R\left(\mu, \mu_{0}, \phi-\phi_{0}\right)}{R_{1}\left(\mu, \mu_{0}, \phi-\phi_{0}\right)}=\frac{\left(\mu+\mu_{0}\right) R\left(\mu, \mu_{0}, \phi-\phi_{0}\right)}{\left(\mu+\mu_{0}\right) R_{1}\left(\mu, \mu_{0}, \phi-\phi_{0}\right)}$

and using Eq. (9) we find

$\lim _{\mu, \mu_{0} \rightarrow 0} R\left(\mu, \mu_{0}, \phi-\phi_{0}\right) / R_{1}\left(\mu, \mu_{0}, \phi-\phi_{0}\right)=1$,

since the right hand side of Eq. (9) is positive. Similarly, we obtain

$\lim _{\mu, \mu_{0} \rightarrow 0} R_{n}\left(\mu, \mu_{0}, \phi-\phi_{0}\right) / R_{1}\left(\mu, \mu_{0}, \phi-\phi_{0}\right)=0, \quad$ for $n>1$.

The ratio of the reflection function to its first order term is an important function, since it is the correction factor to be applied to the easily computed first order term to obtain the reflection function. Numerical studies of this correction factor for homogeneous atmospheres by van de Hulst (1980) have shown that for isotropic scattering the correction factor has a maximum of 8.455 , but that it becomes much smaller for nearly horizontal directions of incidence and reflection. He also found a similar behavior for anisotropic phase functions and mentioned Eq. (15).

\section{Examples}

To illustrate and check the results of the preceding section we will now discuss some simple examples. First for homogeneous and then for inhomogeneous atmospheres. 


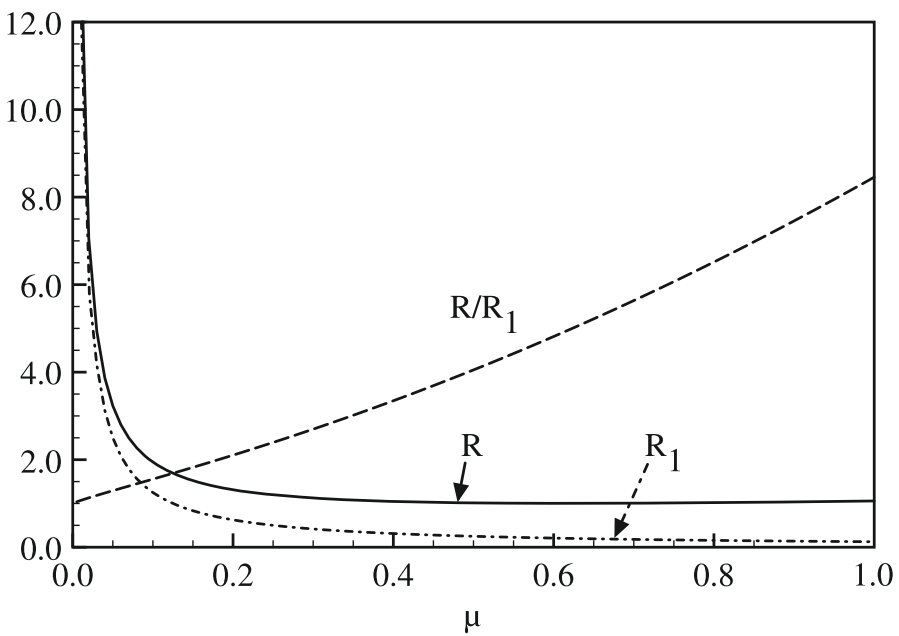

Fig. 2. The reflection function $(R)$ of a non-absorbing, homogeneous, semi-infinite atmosphere with isotropic scattering in case $\mu=\mu_{0}$ is plotted as a function of $\mu$. Also shown are the contribution due to first order scattering $\left(R_{1}\right)$ and the ratio $R(\mu, \mu) / R_{1}(\mu, \mu)$, which in this case equals $8 \mu R(\mu, \mu)$.

\subsection{Homogeneous atmospheres}

Let us consider again the special case of isotropic scattering in a semi-infinite, homogeneous atmosphere with albedo of single scattering $a$. This implies that there is no azimuth dependence for the reflected radiation, so that we can omit $\phi-\phi_{0}$ in equations. The first two orders of scattering of the reflection function can readily be computed by analytic integration over optical depth (Hovenier 1971) or by iteration of an invariance relation (Ambarzumian 1943; Mishchenko et al. 2006). The results are as follows

$$
R_{1}\left(\mu, \mu_{0}\right)=\frac{a}{4\left(\mu+\mu_{0}\right)}
$$

and

$R_{2}\left(\mu, \mu_{0}\right)=\frac{a^{2}}{8\left(\mu+\mu_{0}\right)}\left(k(\mu)+k\left(\mu_{0}\right)\right)$,

where

$k(\mu)=\mu \ln (1+1 / \mu)$.

For the sum over all orders we have (Chandrasekhar 1950)

$R\left(\mu, \mu_{0}\right)=\frac{a}{4\left(\mu+\mu_{0}\right)} H(\mu) H\left(\mu_{0}\right)$,

where $H(\mu)$ is a well-known function, depending on $a$, with $H(0)=1$ (Busbridge 1960). Equations (17)-(20) show that the reflection function and its first two orders of scattering tend to infinity if $\mu$ and $\mu_{0}$ both approach zero. Consequently, this also holds for the multiple scattering component of the reflection function. Clearly Eqs. (17)-(20) are in agreement with Eqs. (5)-(11) and (13)-(16), since $k(0)=0$. Figure 2 shows $R_{1}\left(\mu, \mu_{0}\right)$ and $R\left(\mu, \mu_{0}\right)$ as functions of $\mu$ if $a=1$ and $\mu=\mu_{0}$. Both functions are seen to be strongly increasing when the directions of incidence and reflection tend to horizontal directions.

It follows from Eqs. (17) and (20) that the correction factor

$R\left(\mu, \mu_{0}\right) / R_{1}\left(\mu, \mu_{0}\right)=H(\mu) H\left(\mu_{0}\right)$.

This factor varies between 1 (if $\mu=\mu_{0}=0$ and $a$ is arbitrary) and 8.455 (if $\mu=\mu_{0}=1$ and $a=1$ ). Figure 2 also shows this correction factor in case $\mu=\mu_{0}$.
A lesson to be learned from this simple case is that one should not assume that the discontinuity of the reflection function for horizontal directions of incidence and reflection will always disappear upon integration. Indeed we find from Eq. (17)

$\int_{0}^{1} \mathrm{~d} \mu_{0} R_{1}\left(\mu, \mu_{0}\right)=\frac{a}{4} \ln (1+1 / \mu)$,

which tends to infinity if $\mu$ tends to 0 . So this approach to infinity must also hold if the reflection function is integrated in the same way, since the sum of all orders of the reflection function cannot be smaller than the first order only. Another way to prove this can be obtained from the definition of the $H$-function written in the form

$\int_{0}^{1} \mathrm{~d} \mu_{0} R\left(\mu, \mu_{0}\right)=\frac{1}{2 \mu}(H(\mu)-1)$

and the behavior of the $H$-function when $\mu$ approaches zero (van de Hulst 1980).

Explicit expressions for the reflection function of a semiinfinite or finite homogeneous atmosphere on top of a black surface in terms of functions $H(\mu), X(\mu)$ and $Y(\mu)$ have been published (see e.g. Chandrasekhar 1950) for phase functions that can be written as a sum of a few Legendre polynomials, including Rayleigh scattering. Since $H(0)=1, X(0)=1$ and $Y(0)=0$ (Busbridge 1960) it can readily be verified that in all these cases Eqs. (5)-(11) and (15) are valid.

\subsection{Inhomogeneous atmospheres}

In numerical calculations a vertically inhomogeneous atmosphere is often modeled as a stack of homogeneous layers. Then only the albedo of single scattering and the phase function of the top layer are relevant for the reflected intensity when $\mu$ and $\mu_{0}$ both approach zero. When these values are substituted in Eqs. (4), (5), (7), and (9), Eqs. (4)-(16) are also valid for a stack of homogeneous layers.

Another example of scattering in an inhomogeneous atmosphere is provided by isotropic scattering in a semi-infinite atmosphere with albedo of single scattering given by

$a(\tau)=\exp (-\tau)$

where $\tau$ is the optical depth measured from the top of the atmosphere downwards. In this case we have for $\mu_{0}>0$ (Hovenier \& Stam 2006)

$I_{1}^{\mathrm{t}}\left(\mu, \mu_{0}\right)=\frac{F_{0}}{4} \frac{\mu_{0}}{\left(\mu+\mu_{0}+\mu \mu_{0}\right)}$

so that

$R_{1}\left(\mu, \mu_{0}\right)=\frac{1}{4} \frac{1}{\left(\mu+\mu_{0}+\mu \mu_{0}\right)}$.

It should be noted that the denominators in the last two equations are not simply the sum of $\mu$ and $\mu_{0}$. Equations (25), (26) and the expressions reported by Yanovitskij (1997) for the total intensity of the reflected radiation and the reflection function are in agreement with Eqs. (4)-(16). So the latter equations provide a useful check. 


\section{Related functions}

In addition to the reflection function several alternative functions for describing the reflective properties of a plane-parallel atmosphere are found in the literature. If the difference with the reflection function is only a constant factor all preceding equations must be translated by simply taking this constant into account. But the difference may also involve a function of $\mu$ and/or $\mu_{0}$ and then the translation is less trivial. In such cases one can derive expressions for the alternative function by starting with an expression for the intensity of the reflected light in terms of the alternative function and then working along similar lines as we did for the reflection function or directly use relations for the reflection function to get corresponding results for the alternative function. We give some examples.

Chandrasekhar (1950) defined what he called the scattering function by writing

$I^{\mathrm{t}}\left(\mu, \mu_{0}, \phi-\phi_{0}\right)=\frac{F_{0}}{4 \mu} S\left(\mu, \phi ; \mu_{0}, \phi_{0}\right)$,

so that in view of Eq. (1) the scattering function is related to the reflection function as follows

$S\left(\mu, \phi ; \mu_{0}, \phi_{0}\right)=4 \mu \mu_{0} R\left(\mu, \mu_{0}, \phi-\phi_{0}\right)$.

It follows immediately from this equation and Eq. (8) that

$\lim _{\mu, \mu_{0} \rightarrow 0} S\left(\mu, \phi ; \mu_{0}, \phi_{0}\right)=0$.

So the same must be true for all orders of scattering, since we are dealing here with a sum of nonnegative numbers. Consequently, the orders of scattering of the scattering function, nor the function itself, tend to infinity if we approach the origin of the $\left(\mu_{0}, \mu\right)$ plane along a path given by $\mu=g\left(\mu_{0}\right)$. Using Eq. (9) we find

$$
\begin{aligned}
& \lim _{\mu, \mu_{0} \rightarrow 0}\left(1 / \mu+1 / \mu_{0}\right) S\left(\mu, \phi ; \mu_{0}, \phi_{0}\right)= \\
& \lim _{\mu, \mu_{0} \rightarrow 0}\left(1 / \mu+1 / \mu_{0}\right) S_{1}\left(\mu, \phi ; \mu_{0}, \phi_{0}\right)=a^{\mathrm{t}} Z^{\mathrm{t}}\left(\cos \left(\phi-\phi_{0}\right)\right),
\end{aligned}
$$

which again shows the special role played by single scattering.

Some authors still use the scattering function, but the reflection function is more widely used today. Each one has certain advantages and disadvantages. Hapke (1993) defined several alternatives for the reflection function, most of which differ only by a constant factor from the reflection function. But his so-called bidirectional reflection function $r\left(\mu, \mu_{0}, \phi-\phi_{0}\right)$ has the same peculiar discontinuity as our $I^{\mathrm{t}}\left(\mu, \mu_{0}, \phi-\phi_{0}\right) /\left(\pi F_{0}\right)$.

\section{Azimuth dependence}

So far we have kept the azimuth difference $\phi-\phi_{0}$ fixed and considered what happens when not only the incident radiation tends to the horizontal direction in the $\phi_{0}$-plane but also the reflected radiation tends to the horizontal direction in the $\phi$-plane. More generally, the azimuthal angles may change when $\mu$ and $\mu_{0}$ tend to zero, i.e. $(\mu, \phi)$ may tend to $(0, \bar{\phi})$ and $\left(\mu_{0}, \phi_{0}\right)$ to $\left(0, \bar{\phi}_{0}\right)$ in some way. Clearly, the discontinuities for a plane-parallel atmosphere will then remain and we must only replace $\cos \left(\phi-\phi_{0}\right)$ by $\cos \left(\bar{\phi}-\bar{\phi}_{0}\right)$ in Eqs. (4), (5), (7), and (9). Such situations generally occur in spectrophotometry of planets and satellites when one moves the line of sight to the intensity poles $\left(\mu=\mu_{0}=0\right)$, along the limb $(\mu=0)$, the terminator $\left(\mu_{0}=0\right)$ or an intermediate intensity meridian $\left(\mu=c \mu_{0}\right)$ with finite $c>0$. This must be realized in computations when the outer layers are modeled as a plane-parallel atmosphere (Hovenier \& Stam 2006).
The azimuth dependence of intensities and reflection functions are often handled by making Fourier series expansions, e.g. by writing for an arbitrary function

$$
f\left(\mu, \mu_{0}, \phi-\phi_{0}\right)=f^{0}\left(\mu, \mu_{0}\right)+2 \Sigma_{m=1}^{\infty} f^{m}\left(\mu, \mu_{0}\right) \cos \left(m\left(\phi-\phi_{0}\right)\right),(31)
$$

where the upper index $m$ denotes the Fourier index. In this way we readily find expressions for each Fourier component separately from the equations given in preceding sections. For instance, writing $Z^{\mathrm{t}}\left(0,0, \phi-\phi_{0}\right)$ for the phase function at the top of the atmosphere in case $\mu=\mu_{0}=0$, we obtain for $m \geq 0$ using Eqs. (5) and (7)

$$
\lim _{\mu, \mu_{0} \rightarrow 0} \mu_{0} R^{m}\left(\mu, \mu_{0}\right)=\frac{a^{\mathrm{t}}}{4(c+1)} Z^{\mathrm{t}, m}(0,0)
$$

and

$$
\lim _{\mu, \mu_{0} \rightarrow 0} \mu R^{m}\left(\mu, \mu_{0}\right)=\frac{a^{\mathrm{t}} c}{4(c+1)} Z^{\mathrm{t}, m}(0,0) .
$$

Adding the last two equations gives

$$
\lim _{\mu, \mu_{0} \rightarrow 0}\left(\mu+\mu_{0}\right) R^{m}\left(\mu, \mu_{0}\right)=\frac{a^{\mathrm{t}}}{4} Z^{\mathrm{t}, m}(0,0) .
$$

The azimuthal average of the phase function is positive, since we have assumed that the phase function itself is positive. Hence, the $m=0$ component of the phase function is positive and it follows from Eq. (34) that

$$
\lim _{\mu, \mu_{0} \rightarrow 0} R^{0}\left(\mu, \mu_{0}\right)=\infty
$$

However, $Z^{\mathrm{t}, m}(0,0)$ can be positive, negative or zero if $m>0$. Consequently, if $\mu$ as well as $\mu_{0}$ tend to zero the limit of $R^{m}\left(\mu, \mu_{0}\right)$ may be plus infinity, minus infinity or it may be impossible to determine this limit from Eq. (34). This may be illustrated by considering the following simple cases.

(i) The phase function

$$
Z^{t}(\cos \Theta)=1-\frac{1}{2} \cos \Theta
$$

has

$Z^{\mathrm{t}, 0}(0,0)=1$

and

$Z^{\mathrm{t}, 1}(0,0)=-1 / 4$

(ii) Rayleigh's phase function, i.e.

$Z^{\mathrm{t}}(\cos \Theta)=\frac{3}{4}\left(1+\cos ^{2} \Theta\right)$

has

$Z^{\mathrm{t}, 0}(0,0)=9 / 8$

$Z^{\mathrm{t}, 1}(0,0)=0$

$Z^{\mathrm{t}, 2}(0,0)=3 / 16$.

Using the explicit expressions (Chandrasekhar 1950) for the reflected intensity of a homogeneous semi-infinite atmosphere with $a=1$ for these two phase functions one can easily find that Eqs. (32)-(34) are satisfied. 


\section{Approximations}

Accurate computations of the reflection function of a planeparallel atmosphere are generally not easy and also laborious, especially when results are needed for many model parameters. Therefore, it is not surprising that a variety of approximative formulae have been proposed (Sobolev 1975; van de Hulst 1980; Kokhanovsky 2001). Here we wish to point out that at least some of these are not realistic for directions of incidence and reflection that are both nearly horizontal.

For instance, according to the popular Lambert's reflection law the reflection function is a constant not larger than one for all directions of incidence and reflection. So this reflection function does not obey Eq. (9) and can never tend to infinity, which is in conflict with Eq. (11).

Another example is the so-called "rapid-guess formula" of van de Hulst (1980) for a non-absorbing homogeneous semiinfinite atmosphere

$R^{0}\left(\mu, \mu_{0}\right)=1+p(1-3 \mu / 2)\left(1-3 \mu_{0} / 2\right)$,

where the upper index, 0 , refers to the azimuth independent term and $p$ is a finite constant. This formula clearly violates Eq. (35) and, therefore, may give unacceptable errors for nearly horizontal directions of incidence and reflection. To show this we consider isotropic scattering for which the value $p=0.4$ was recommended by van de Hulst (1980). Using this value in the rapid-guess formula with $\mu=\mu_{0}=0.1$ gives for the reflection function 1.289 , which is $33.72 \%$ too low, since accurate multiple scattering calculations give 1.94485 . For smaller values of $\mu$ and $\mu_{0}$ the errors in the reflection function are still larger. Integration of the reflection function as in Eq. (23) gives for $\mu=0.1$ the accurate value of 1.23675 , but Eq. (43) gives 1.0850 which is still $12.27 \%$ too low.

Finally, we mention a formula that is often used for a homogeneous atmosphere above a black surface when the optical thickness $b$ is a small positive number, namely

$R\left(\mu, \mu_{0}, \phi-\phi_{0}\right)=\frac{a b}{4 \mu \mu_{0}} Z(\cos \Theta)$,

where

$\cos \Theta=-\mu \mu_{0}+\sqrt{\left(1-\mu^{2}\right)\left(1-\mu_{0}^{2}\right)} \cos \left(\phi-\phi_{0}\right)$.

Although Eq. (44) does not violate Eq. (11) the approach of this approximate reflection function to infinity if $\mu$ and $\mu_{0}$ both tend to zero is apparently not correct, since Eqs. (5) and (7)-(9) are not satisfied.

Consequently, if $\mu$ and $\mu_{0}$ are very small one should be careful with using approximations for the reflection function. Fortunately, the results of the preceding sections suggest that it may then be sufficient to compute only a few orders of scattering (see e.g. Hovenier 1971; van de Hulst 1980; Mishchenko et al. 2006) instead of a more laborious complete multiple scattering calculation. This holds in particular when the optical thickness and/or the albedo of single scattering is not large. For example, for isotropic scattering in a homogeneous semi-infinite atmosphere with $a=0.4$ and $\mu=\mu_{0}=0.1$ the sum of the first two orders of scattering of the reflection function is only $1.6 \%$ too low and the sum of the first three orders even less than $0.34 \%$.

\section{Concluding remarks}

Numerous complicated equations occur in the theory of multiple light scattering in homogeneous and inhomogeneous plane-parallel atmospheres (see e.g. Chandrasekhar 1950; Sobolev 1975; van de Hulst 1980; Yanovitskij 1997; Hovenier et al. 2004; Mishchenko et al. 2006). Since there is always a possibility that printed equations contain errors and their derivations are not always given, it is useful to have simple checks available like letting $\mu$ and $\mu_{0}$ approach zero and comparing the results with expressions in this paper.

On performing model computations it is usually very helpful to know and understand what happens with the reflection function or a related function in limiting cases (Irvine 1983). This kind of knowledge is provided in this paper even for a complicated model of an inhomogeneous atmosphere with an arbitrarily reflecting surface underneath. In particular one should be prudent in the proximity of discontinuities like those presented in this paper.

We have shown that a discontinuity exists for the reflection function which may hamper interpolation and extrapolation. This problem may be by-passed by multiplying the reflection function by a simple function of $\mu$ and $\mu_{0}$, like $\mu+\mu_{0}$ (cf. Eq. (9)), before performing the interpolation or extrapolation (Knibbe et al. 2000). This is illustrated in Fig. 2 by the curve marked $R / R_{1}$ since in the case considered this curve equals $8 \mu R(\mu, \mu)$.

We have also shown that great care should be exercised with using approximative formulae for the reflection function, since they may lead to large errors for nearly horizontal directions. This holds, for instance, for the "rapid guess formula" and the very popular Lambert reflection law, which is often used, e.g. for cloudy atmospheres of planets (see e.g. Kokhanovsky 2006). Although approximative formulae exist that do not give large errors for nearly horizontal directions, more accurate results are possibly obtained by computing a few orders of scattering for such directions.

\section{References}

Ambarzumian, V. A. 1943, Dokl. Akad. Nauk. S.S.S.R, 38, 229

Busbridge, L. 1960, The Mathematics of Radiative Transfer (London: Cambridge University Press)

Chandrasekhar, S. 1950, Radiative transfer (Oxford: Clarendon Press)

Courant, R. 1962, Differential and Integral Calculus, 2nd edition (London: Blackie and Son)

Hansen, J. E., \& Travis, L. D. 1974, Space Sci. Rev., 16, 527

Hapke, B. 1993, Theory of reflectance and emittance spectroscopy, Topics in Remote Sensing (Cambridge, UK: Cambridge University Press)

Hovenier, J. W. 1971, A\&A, 13, 7

Hovenier, J. W., \& Stam, D. M. 2006, J. Quant. Spectr. Radiat. Transf., 101, 1 Hovenier, J. W., \& Stam, D. M. 2007, J. Quant. Spectr. Radiat. Transf., 107, 83

Hovenier, J. W., van der Mee, C., \& Domke, H. 2004, Transfer of Polarized Light in Planetary Atmospheres, Basic Concepts and Practical Methods (Dordrecht: Kluwer, Berlin: Springer)

Irvine, W. M. 1983, Icarus, 55, 187

Knibbe, W. J. J., de Haan, J. F., Hovenier, J. W., et al. 2000, J. Quant. Spectr. Radiat. Transf., 64, 173

Kokhanovsky, A. A. 2001, Optics of Light Scattering Media (Chicester: Springer, Praxis)

Kokhanovsky, A. A. 2006, Cloud Optics (Berlin: Springer)

Minnaert, M. 1935, Physica, 2, 363

Mishchenko, M. I., Dlugach, Z. M., Yanovitskij, E. G., \& Zakharova, N. T. 1999, J. Quant. Spectr. Radiat. Transf., 63, 409

Mishchenko, M. L., Travis, L. D., \& Lacis, A. A. 2006, Multiple Scattering of Light by Particles (Cambridge: University Press)

Sobolev, V. V. 1975, Light scattering in planetary atmospheres (Oxford and New York: Pergamon Press)

van de Hulst, H. C. 1980, Multiple Light Scattering, Tables, Formulas, and Applications, Vols. 1 and 2 (New York: Academic Press)

Yanovitskij, E. G. 1997, Light Scattering in Inhomogeneous Atmospheres (Berlin: Springer) 